\title{
Modelo Pélvico Sintético como uma Ferramenta Didática Efetiva Comparada à Pelve Cadavérica
}

\author{
A Synthetic Three-Dimensional Pelvic Model \\ as an Effective Didactic Tool Compared to \\ Cadaveric Pelvis
}

\author{
Hélio Sérgio Pinto Portugal \\ Paulo Cesar Rodrigues Palma \\ Rogério de Fragall \\ Cássio Luis Zanettini Riccetto \\ Sergio Rocha ${ }^{I I}$ \\ Luciane Carias
}

PALAVRAS-CHAVE

- Educação Médica.

- Anatomia.

- Modelo Anatômico.

- Soalho Pélvico.

\section{KEYWORDS}

- Medical Education.

- Anatomy.

- Anatomical Model.

- Pelvic Floor

Recebido em: 07/12/2010

Reencaminhado em: 25/05/2011

Aprovado em: 08/09/2011

REVISTA BRASILEIRA DE EDUCAÇ̄̃o MÉDICA 50235 (4): $502: 506: 2011$
${ }^{I}$ Universidade Estadual de Campinas, Campinas, SP, Brasil.

"I Universidade Federal do Paraná, Curitiba, PR, Brasil.

\section{RESUMO}

A anatomia é considerada matéria essencial à educação médica. Aulas práticas com uso de componentes anatômicos manipuláveis são fundamentais ao ensino è à aprendizagem. O objetivo deste estudo foi avaliar o Modelo Sintético de Pelve (Masp) como ferramenta didática, comparada à pelve cadavérica (PC) tradicionalmente utilizada, bem como a satisfação dos estudantes em relação a ambos os métodos. Sessenta e sete estudantes receberam aula teórica após teste teórico preliminar (TTP). Foram randomizados em três grupos: G1 teve aula prática tradicional (APT); G2, aula prática com Masp (APM); e G3 não teve aula prática. Um teste final (TTF) foi aplicado a todos os grupos. G1 e G2 submeteram-se à avaliação do método (AM). A análise estatística foi realizada utilizando-se Anova (Análise de Variância) e teste não paramétrico Mann-Whitney. No TTF, G3 apresentou escores mais baixos do que G1 $(p=0,041)$ e G2 ( $p=0,000)$. Não foi encontrada diferença estatisticamente significante entre G1 e G2 ( $p>0,05)$. G2 apresentou maior satisfação com o método $(p=0,001)$. Concluiu-se que PC e Masp provaram ser ferramentas didáticas efetivas e que G2 mostrou maior satisfação.

Anatomy is considered a fundamental subject of medical education. Practical classes which involve the use of manipulable anatomic components are essential to teaching and learning. The aim of this study was to evaluate the synthetic pelvic model (SPM) as a didactic tool, compared to the traditionally-used cadaveric pelvis (CP), as well as student satisfaction in relation to both methods. Sixty-seven students were given a theory class following the preliminary theoretical test (PTT). The study sample was randomized in three groups: G1 had a traditional practical class (TPC); G2 had a practical class with SPM (PCS); and G3 had no practical class. A final test (FTT) was applied to all the groups. G1 and $G 2$ were subjected to method evaluation $(M E)$. Statistical analysis was conducted using analysis of variance (ANOVA) and the Mann-Whitney U test. In the FTT, G3 presented scores lower than G1 $(p=0.041)$ and G2 ( $p=0.000)$. No statistically significant difference was found between G1 and G2 $(p>0.05)$. G2 presented greater satisfaction with the method ( $p=0.001)$. In conclusion, both CP and SPM proved to be effective didactic tools and student satisfaction was greater with G2. 


\section{INTRODUÇÃO}

A anatomia tem, sem dúvida, a mais longa história como disciplina no ensino médico formalizado ${ }^{1}$. O conhecimento das estruturas anatômicas do assoalho pélvico feminino é crucial para a compreensão e o entendimento satisfatórios nas diversas áreas da medicina, especialmente em uroginecologia.

É essencial que exista, além do conhecimento anatômico, um claro entendimento das funções de cada elemento anatômico desta região, sítio de intrincadas interações entre suas várias estruturas. Uma vez entendida esta relação entre os elementos anatômicos, torna-se claro que não existem elementos estáticos ou independentes nesse segmento da pelve feminina.

A pelve óssea, os músculos do assoalho pélvico e as estruturas anorretais associadas compreendem uma das mais complexas regiões da anatomia humana. O estudo do assoalho pélvico é difícil por diversas razões. Primeiramente, esta região, com frequência, é inacessível porque está cercada pelos ossos pélvicos. Este espaço relativamente pequeno também contém muitos sistemas orgânicos, e determinadas estruturas são observadas somente através de dissecações especiais realizadas com sacrifício de outras estruturas ${ }^{2}$. Adicionalmente, durante a cirurgia ou dissecação cadavérica, a sintopia difere do estado normal, pela alteração do tônus dos músculos.

Inevitavelmente, o estudo da anatomia do assoalho pélvico feminino encontra dificuldade de aprendizado. Isto se deve à complexidade das estruturas envolvidas e à sobreposição dos elementos anatômicos na cavidade pélvica, especialmente no material cadavérico.

O estudo da anatomia do assoalho pélvico a partir de figuras bidimensionais não é satisfatoriamente adequado para elucidá-lo porque não fornece uma percepção tridimensional dos elementos anatômicos. Existem diversos modelos sintéticos para o estudo do assoalho pélvico, porém eles apresentam elementos anatômicos fundidos. Este tipo de ferramenta didática representa uma barreira para um raciocínio anatômico completo, conduzindo ao ensino e aprendizagem deficientes.

Um Modelo Anatômico Sintético de Pelve (Masp) foi desenvolvido com o objetivo de se tornar um instrumento para incrementar o modo de ensino e aprendizado desse complexo segmento da anatomia. A intenção desse modelo não é mover o cadáver do lugar que este tem como a principal ferramenta para o estudo da anatomia, mas prover um material suplementar em que os elementos anatômicos sejam claramente distinguíveis e manipuláveis.

O Masp é constituído por peças de etileno acetato de vinila, que é um copolímero, além de látex, Velcro® e malha metá- lica. Estas peças foram moldadas de acordo com cada elemento anatômico, distinguindo-se o tipo de tecido representado, sendo as peças ajustadas na pelve óssea feminina.

Trabalhamos com a hipótese de que o Masp é uma ferramenta didática efetiva para o estudo do assoalho pélvico feminino, comparada à pelve cadavérica.

O objetivo deste estudo foi avaliar o Masp como instrumento didático em comparação à aula tradicional de anatomia, em que se utiliza a pelve cadavérica (PC), bem como a satisfação dos estudantes em relação a ambos os métodos.

\section{MATERIAIS E MÉTODOS}

Este foi um estudo experimental, prospectivo aplicado, e sua abordagem metodológica foi hipotético-dedutiva.

A aprovação ética para este estudo foi recebida do Comitê de Ética da Universidade Federal do Paraná e cada voluntário forneceu consentimento informado antes de sua participação.

Sessenta e sete estudantes de graduação em Medicina foram recrutados para este estudo. O critério de exclusão para participar do estudo foi a incapacidade, momentânea ou definitiva, para manipular os elementos anatômicos.

\section{Instrumentos de avaliação}

Como instrumentos de avaliação foram utilizados os seguintes questionários: I - Teste Teórico Preliminar (TTP), para verificação do conhecimento prévio em relação à anatomia pélvica; II - Teste Teórico Final (TTF), para verificação do conhecimento agregado após as aulas; III - Avaliação do Método (AM), para verificar a satisfação dos estudantes em relação ao componente anatômico utilizado na aula prática (PC ou Masp). Nesta última avaliação foi utilizada uma escala visual analógica na qual "zero" estava relacionado a nenhuma satisfação e "dez" à satisfação plena. TTP e TTF foram constituídos do mesmo questionário, cujos momentos de aplicação determinaram denominações diferentes aos testes. O conteúdo de ambos foi um questionário com uma sequência de 20 questões que exigiam a resposta "verdadeira" ou "falsa". Estas questões foram relacionadas aos elementos anatômicos do assoalho pélvico feminino, tais como músculos, tendões, ossos, fáscias, nervos, arcos tendíneos e órgãos.

\section{Ferramentas didáticas comparativas}

Para este estudo, que compara duas ferramentas didáticas para o ensino e aprendizagem da anatomia pélvica, especialmente dos componentes do assoalho pélvico, foram utilizados uma pelve cadavérica feminina (Figura 1A) e um modelo pélvico sintético feminino (Masp) (Figura 1B). 


\section{FigURA 1}

Pelve Cadavérica - PC (A) e Modelo Anatômico Sintético de Pelve - Masp (B)

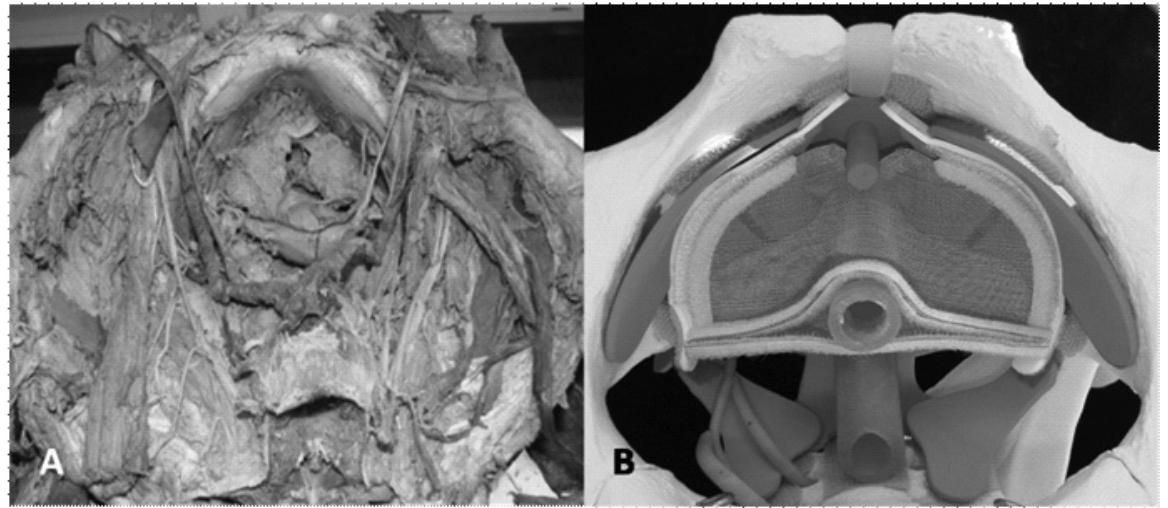

\section{Coleta de Dados}

O TTP foi aplicado a todos os grupos simultaneamente. Os estudantes foram então divididos em três grupos, G1, G2 e G3, de acordo com as três séries numéricas, sorteadas aleatoriamente. Os grupos G1 e G2 assistiram a aulas de anatomia com diferentes métodos didáticos: aula prática tradicional de anatomia (APT) para G1, com um modelo de assoalho pélvico sintético comum, incluindo a utilização de atlas anatômicos, além de quatro peças cadavéricas de pelve feminina e pelve óssea cadavérica; aula prática de anatomia (APM) com quatro modelos Masp para o G2, sem utilização de outros recursos, como atlas e tutoriais. O TTF foi aplicado a todos os grupos.
Os grupos G1 e G2 foram submetidos à AM (ver detalhes na Figura 2).

\section{Análise Estatística}

A análise estatística foi realizada utilizando-se Anova multivariada para comparar os diferentes métodos didáticos, tradicional e proposto, em relação ao aprendizado, após confirmação da normalidade da amostra através do teste de homogeneidade de Levene $(p \geq 0,05)$. O teste não paramétrico Mann-Whitney foi utilizado para comparar a satisfação relacionada aos métodos, por seus recursos didáticos. O nível de significância foi determinado como $p \leq 0,05$.

FIGURA 2

Fluxograma do estudo

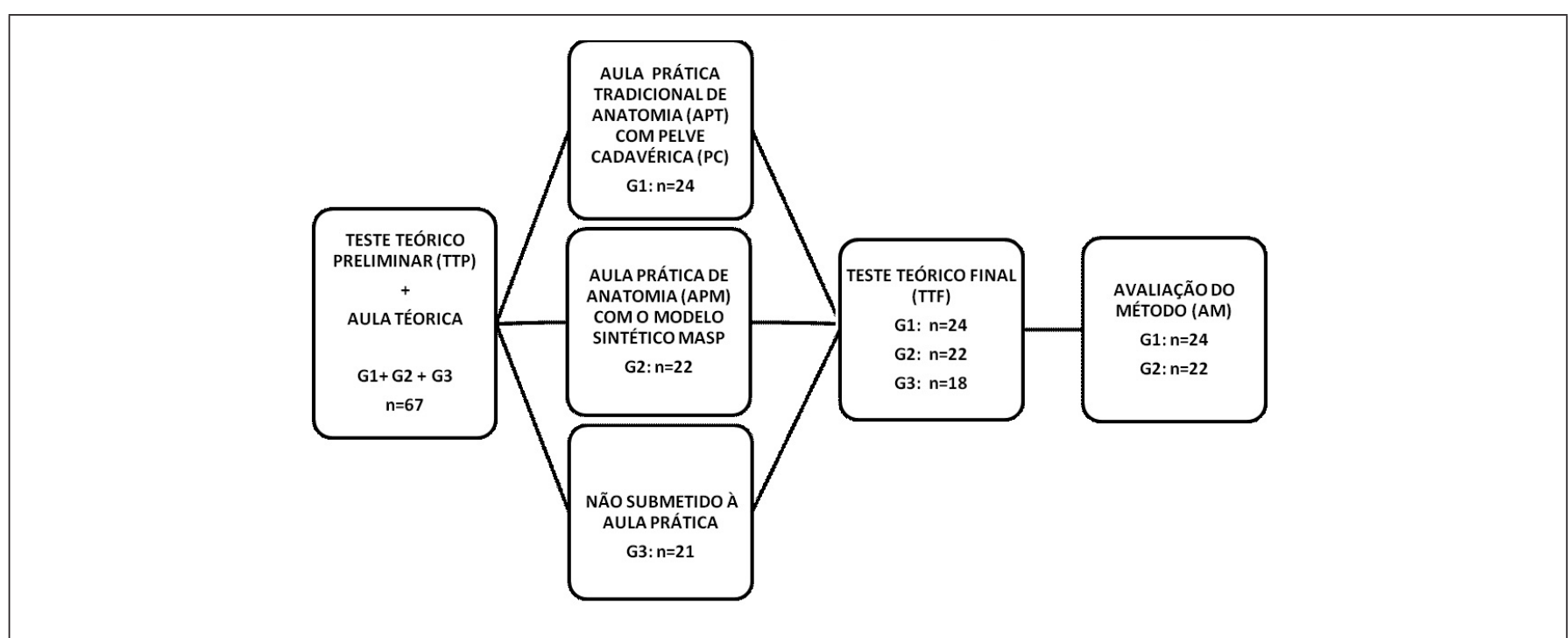




\section{RESULTADOS}

A idade média foi 19,41 $( \pm 1,58)$ em G1, 19,31 $( \pm 3,43)$ em G2 e $19,42( \pm 3,35)$ em G3. Diferenças não significantes foram observadas entre os grupos na primeira avaliação (TTP) $(p>0,05)$. Diferentemente, na avaliação após as aulas (TTF), os estudantes de G3 apresentaram escores mais baixos que os de G1 $(p=0,041)$ e G2 $(p=0,000)$ (Figura 3).

\section{FIGURA 3}

Respostas corretas (média e desvio padrão) por grupo no Teste Teórico Preliminar (TTP) e final (TTF). Embora G2 tenha apresentado maior escore do que G1, tal diferença não foi estatisticamente significante ( $p>0,05)$. G3 apresentou escores mais baixos em relação a G1 ( $\left.p=0,041^{*}\right)$ e G2 $\left(p=0,000^{* *}\right)$.

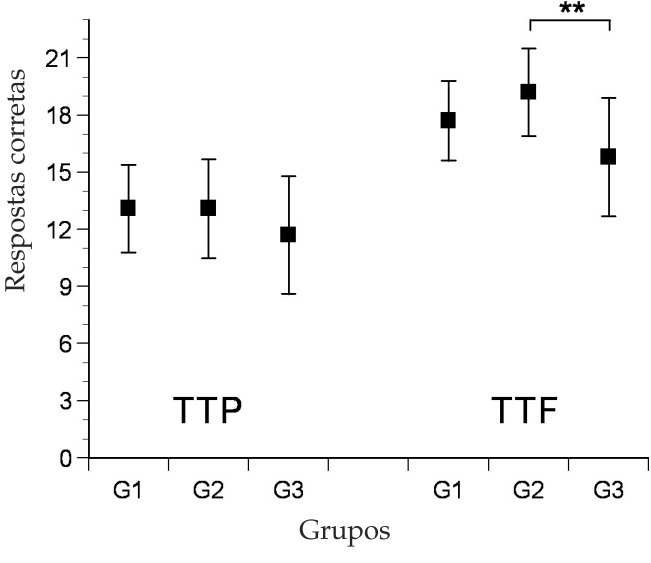

FigURA 4

Grau de satisfação dos estudantes dos grupos G1 e G2 com o método utilizado nas aulas práticas. Foi encontrada diferença estatisticamente significante $(p=0,001)$, sendo que G2 mostrou mais satisfação com o método.

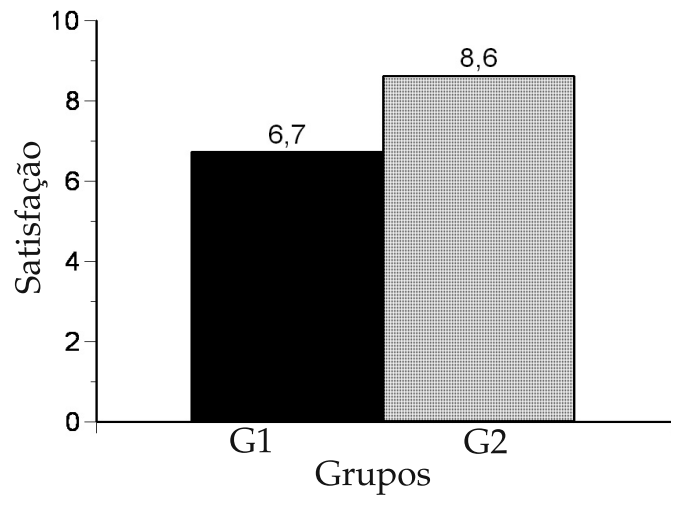

Não foi encontrada diferença estatisticamente significante entre G1 e G2 no TTF ( $p>0,05)$. Entretanto, G2 mostrou estar mais satisfeito com o método utilizado na aula prática ( $p=$ 0,001) (Figura 4).

\section{DISCUSSÃO}

Embora não haja diferença estatisticamente significante, G2 mostrou escores maiores do que G1 no TTF. Neste estudo, PC e Masp provaram ser instrumentos didáticos efetivos para o estudo do assoalho pélvico feminino quando utilizados em aulas práticas de anatomia. Além disto, G2 mostrou maior grau de satisfação com o método utilizado na aula prática.

Estes achados apoiam a hipótese de que o Masp é uma ferramenta didática efetiva, segundo o propósito para o qual foi desenvolvido. Este estudo mostrou ainda que, para o estudo do assoalho pélvico feminino, somente a aula teórica não fornece o conhecimento anatômico suficiente e que a aula prática é indispensável.

Não foram encontradas na literatura comparações entre os modelos sintéticos e o material cadavérico como ferramentas didáticas. Um estudo recente, porém, utilizou o Masp e relatou que este permitiu o entendimento e a prática de várias técnicas cirúrgicas, substituindo modelos animais, reconstruções tridimensionais computadorizadas e imagens bidimensionais, contribuindo de forma notável para a brevidade da curva de aprendizado ${ }^{3}$.

Numa pesquisa com 112 anatomistas profissionais, Patel e Moxham ${ }^{4}$ observaram que a preferência pelos métodos de ensino, em ordem descendente, era: dissecação cadavérica, ensino prático com uso da prossecução, ensino baseado na anatomia radiológica viva, ensino eletrônico através de computador, palestras e aulas didáticas em classe, e uso de modelos. Diferentemente daquela pesquisa, este estudo avaliou os instrumentos didáticos a partir dos estudantes, pelo conhecimento anatômico agregado ao prévio, mas não avaliou os professores e seus métodos preferidos.

Entendemos que o Masp é equivalente à PC com relação à característica de seus potenciais didáticos. Além disto, o Masp proporcionou maior satisfação aos estudantes.

O material cadavérico é a ferramenta de primeira escolha para o processo de ensino e aprendizado, mas há desafios para reintegrar as mais efetivas ferramentas de ensino e aprendizagem enquanto se mantêm os valores benéficos da dissecação ortodoxa $^{5}$

A manipulação de cadáveres para produzir novos pontos de vista usualmente não é muito prática e, muitas vezes, é impossível ${ }^{6}$. Embora diversos autores declarem que a dissecação do assoalho pélvico e o entendimento de suas estruturas são difíceis ${ }^{2,7}$, não foram encontrados estudos similares que apresentassem outros recursos para incrementar este entendimento, como modelos físicos do assoalho pélvico feminino que 
utilizem material sintético. Isto se refere a modelos manipuláveis, cujas peças pudessem detalhar importantes elementos estruturais anatômicos, como fáscias e arcos tendíneos, além de músculos, tendões, vasos, nervos e órgãos, proporcionando ao estudante um instrumento alternativo de aprendizado.

O resultado deste estudo preliminar deixa algumas questões não respondidas. O experimento testou o aprendizado e a recordação imediatos e não avaliou os efeitos do aprendizado a longo prazo. A maior limitação deste estudo foi a inexistência de um quarto grupo que fosse submetido a ambas as aulas práticas, utilizando PC e Masp. Poderia haver ainda um quinto grupo que se submetesse às duas aulas de modo inverso, primeiramente com Masp e em seguida com PC. Isto certamente proporcionaria uma resposta completa em relação à real satisfação com os métodos. Esta limitação acorreu principalmente devido a limitações de tempo e número de estudantes.

Para a aplicação da APT com PC, o professor utilizou três peças anatômicas (pelves femininas) e um modelo sintético comum de pelve feminina contendo um assoalho pélvico com poucas divisões, além de tutoriais como atlas de anatomia. Isto ocorreu porque os autores não quiseram interferir na metodologia da aula prática tradicional ou modificá-la. Entretanto, para a aplicação da APM foram utilizados três Masp e nenhum outro material coadjuvante.

É incerto se os achados deste estudo experimental generalizaram todos esses cenários, e isto será mais bem elucidado por pesquisas futuras. Apesar destas limitações, neste momento parece interessante que educadores e estudantes de Medicina considerem cuidadosamente as características inerentes de certos materiais instrucionais sintéticos. Estes podem se diferenciar de outros quanto ao potencial didático, uma vez que o eficiente uso de nova tecnologia e métodos de ensino deve permitir melhor ensino e compreensão da anatomia ${ }^{8}$

Como Mayer ${ }^{9}$ declarou, no mundo científico, modelos são frequentemente utilizados para explicar ideias complexas e parecem mudar o foco e a organização do pensamento científico. O presente estudo parece corroborar esta posição, uma vez que o Masp explicou claramente o assoalho pélvico tanto quanto o fez a PC.

\section{CONCLUSÕES}

Este estudo concluiu que a PC e o Masp se mostraram ferramentas didáticas efetivas para o estudo do assoalho pélvico feminino quando utilizados em aulas de anatomia prática. O Masp parece ter sido um recurso didático que produziu satisfação nos estudantes pelo seu emprego em aula prática de anatomia. Outras pesquisas são necessárias para avaliar se o Masp, como uma ferramenta didática alternativa ou suplementar, pode garantir satisfatoriamente seu propósito em aplicações de longo tempo.

\section{REFERÊNCIAS}

1. McLachlan JC, Patten D. Anatomy teaching: ghosts of the past, present and future. Med Educ. 2006;40:243-53.

2. Smith WC. The levator ani muscle: its structure in man, and its comparative relationships. Anat Rec. 1923;26:175-203.

3. Palma P, Riccetto C, Fraga R, Portugal HSP, Dambros M, Rincón ME, Silveira A, Netto NR Jr. Tridimensional anatomy and virtual surgery for transobturator procedures. Actas Urol Esp. 2007;31(4):361-5.

4. Patel KM, Moxham BJ. Attitudes of professional anatomists to curricular change. Clin Anat. 2006;19:132-41.

5. Sugand K, Abrahams P, Khurana A. The anatomy of anatomy: a review for its modernization. Anat Sci Educ. 2010;3: 83-93.

6. Hisley KC, Anderson LD, Smith SE, Kavic SM, Tracy JK. Coupled physical and digital cadaver dissection followed by a visual test protocol provides insights into the nature of anatomical knowledge and its evaluation. Anat Sci Ed. 2008;1:27-40.

7. B SN, Rodenbaugh DW. Modeling the anatomy and function of the pelvic diaphragm and perineal body using a "string model". Advan Physiol Educ. 2008;32:169-70.

8. Collins JP. Modern approaches to teaching and learning anatomy. BMJ. 2008;337:a1310.

9. Mayer RE. Models for understanding. Rev Educ Res. 1989;59:43-64.

\section{CONTRIBUIÇÃO DOS AUTORES}

Helio Sérgio Pinto Portugal: autor principal e confeccionador do artigo; Paulo Cesar Rodrigues Palma: colaborou na elaboração da metodologia; Rogério de Fraga: orientou a confecção do artigo em todas as fases de sua construção; Cássio Luis Zanettini Riccetto: participou das discussões metodológicas e auxiliou no tratamento estatístico; Sérgio Rocha: revisou os princípios anatômicos; Luciane Carias: revisou os princípios anatômicos e colaborou na revisão do tratamento estatístico.

\section{CONFLITO DE INTERESSES}

Declarou não haver.

\section{ENDEREÇO PARA CORRESPONDÊNCIA}

Helio Sérgio Pinto Portugal

Universidade Estadual de Campinas, Faculdade de Ciências Médicas, Depto Cirurgia.

Cidade Universitária Zeferino Vaz

Barão Geraldo - Campinas

13083-970 Cx. Postal 6111 SP

E-mail: hspportugal@gmail.com 